\title{
How Do Patients with Newly Diagnosed SLE Present? A Multicenter Cohort of Early SLE to Inform the Development of New Classification Criteria
}

Mosca $\mathrm{M}^{1}$, Costenbader $\mathrm{KH}^{2}$, Johnson $\mathrm{SR}^{3}$, Lorenzoni $\mathrm{V}^{4}$, Sebastiani $\mathrm{GD}^{5}$, Piga $\mathrm{M}^{6}$, Hoyer $\mathrm{BF}^{7}$, Navarra $\mathrm{S}^{8}$, Bonfa ${ }^{9}$, Ramsey-Goldman $\mathrm{R}^{10}$, Medina-Rosas ${ }^{3}$, Tani $\mathrm{C}^{1}$, Tedeschi $\mathrm{SK}^{2}$, Dörner $\mathrm{T}^{11}$, Aringer $\mathrm{M}^{12}$, Touma $\mathrm{Z}^{3}$

On behalf of the ACR/EULAR group; New SLE classification criteria for clinical research are being developed, sponsored by EULAR and ACR

${ }^{1}$ University of Pisa, Pisa, Italy

${ }^{2}$ Harvard Medical School, Boston, United States

${ }^{3}$ Division of Rheumatology, Department of Medicine; Institute of Health Policy, Management and Evaluation, University of Toronto, Toronto, Canada

${ }^{4}$ Scuola Superiore Sant'Anna, Pisa, Italy

${ }^{5}$ Azienda Ospedaliera San Camillo-Forlanini, Roma, Italy

6 Università di Cagliari

${ }^{7}$ Department of Medicine I, Christian-Albrechts-University and University Hospital SchleswigHolstein, Kiel, Germany

${ }^{8}$ University of Santo Tomas, Manila, Philippines

${ }^{9}$ Hospital das Clinicas HCFMUSP, Faculdade de Medicina, Universidade de Sao Paulo, Sao Paulo, SP, Brazil

${ }^{10}$ Northwestern University, Chicago IL

${ }^{11}$ Department of Medicine/Rheumatology and Clinical Immunology, Charité University Medicine Berlin and DRFZ, Berlin, Germany

${ }^{12}$ University Medical Center and Faculty of Medicine Carl Gustav Carus, TU Dresden, Dresden, Germany

Marta Mosca, MD, PhD

University of Pisa, Pisa,Italy

Email: marta.mosca@med.unipi.it

Karen H Costenbader, MD, MPH

Brigham and Women's Hospital

Harvard Medical School, Boston, United States

Email: KCOSTENBADER@bwh.harvard.edu

\section{Sindhu R. Johnson MD PhD}

Division of Rheumatology, Department of Medicine; Institute of Health Policy, Management and Evaluation, University of Toronto, Toronto, Canada

Email: Sindhu.Johnson@uhn.ca

Valentina Lorenzoni, PhD

Institute of Management, Scuola Superiore Sant'Anna, Pisa, Italy

Email: valentina.lorenzoni@santannapisa.it

Gian Domenico Sebastiani, MD

Azienda Ospedaliera San Camillo-Forlanini, Roma, Italy

Email: giandoreum@libero.it 


\section{Alessandro Mathieu}

<amath@unica.it>

\section{Bimba Hoyer, MD}

Department of Medicine I, Christian-Albrechts-University and University Hospital Schleswig-

Holstein, Kiel, Germany

Email: Bimba.Hoyer@charite.de

\section{Sandra Navarra, MD}

University of Santo Tomas, Manila, Philippines

Email: sandra_navarra@yahoo.com

\section{Eloisa Bonfa, MD}

Hospital das Clinicas HCFMUSP, Faculdade de Medicina, Universidade de Sao Paulo, Sao Paulo, SP, Brazil

Email: eloisa.bonfa@hc.fm.usp.br

\section{Rosalind Ramsey-Goldman, MD, DrPH}

Northwestern University Feinberg School of Medicine, Chicago IL

Email: rgramsey@ northwestern.edu

Jorge Medina-Rosas, MD

Toronto Western Hospital, Centre for Prognosis Studies in the Rheumatic Diseases, University of Toronto Lupus Clinic, Toronto, Ontario, Canada

Email: kikemedina@gmail.com

Chiara Tani, MD, PhD

University of Pisa, Pisa, Italy

Email:chiara.tani@for.unipi.it

\section{Sara K. Tedeschi, MD, MPH}

Brigham and Women's Hospital

Harvard Medical School, Boston, United States

Email: stedeschi1@bwh.harvard.edu

\section{Thomas Dörner, MD}

Department of Medicine/Rheumatology and Clinical Immunology, Charité University Medicine Berlin and DRFZ, Berlin, Germany

Email: Thomas.Doerner@charite.de

Martin Aringer, MD

University Medical Center and Faculty of Medicine Carl Gustav Carus, TU Dresden, Dresden, Germany

Email: Martin.Aringer@uniklinikum-dresden.de

\section{Zahi Touma, MD, PhD}

Division of Rheumatology, Department of Medicine; Institute of Health Policy, Management and Evaluation, University of Toronto, Toronto, Canada

Email: Zahi.Touma@uhn.ca 


\title{
Corresponding author:
}

Marta Mosca, MD,PhD

University of Pisa, Pisa,Italy

Email: marta.mosca@med.unipi.it

\begin{abstract}
Background

Systemic lupus erythematosus (SLE) presents with myriad non-specific signs and symptoms also found in other medical conditions. This international study aimed to compare early SLE manifestations to those of diseases mimicking SLE.
\end{abstract}

\section{Methods}

Clinical and laboratory data of patients diagnosed within 12 months of SLE were collected at 3 North American, 2 European, 1 Asian and 1 South American academic lupus centers. Similar data were collected for patients presenting for possible SLE, but not diagnosed with SLE. Clinical and serological manifestations were compared between the two groups. Performance of the 1997 ACR and 2012 SLICC SLE classification criteria was also tested.

\section{Results}

Of 616 patients, 389 had early SLE and 227 mimicking conditions. Unexplained fever was more common in early SLE than mimicking conditions (34.5\% vs. $13 \%, \mathrm{p}<0.001)$. Features less common in SLE than mimicking conditions included Raynaud's phenomenon $(22.1$ vs. $48.5 \%, \mathrm{p}<0.001)$, sicca symptoms $(4.4 \%$ vs. $34.4 \%)$, dysphagia $(0.3 \%$ vs. $6.2 \%, \mathrm{p}<0.001)$, and fatigue $(28.3 \%$ vs. $37.0 \%, \mathrm{p}=0.024)$. ACR and SLICC criteria items were significantly more frequent among early SLE patients. At diagnosis, 33.9\% of early SLE patients did not fulfill ACR 1997 criteria (sensitivity 66.1\%). The SLICC criteria missed fewer (16.5\%) early SLE patients than the ACR criteria (33.9\%), but the latter had higher specificity (91.6\% vs. $82.4 \%)$.

\section{Conclusions}

In this multicenter cohort, clinical manifestations that could help to distinguish early SLE from mimicking conditions were identified. These findings could aid in earlier SLE diagnosis and provide information for ongoing initiatives to revise SLE classification criteria.

Word count: 249

Short title: Manifestations of early SLE

Key words: Systemic lupus erythematosus; Classification; SLE; Early 


\section{Introduction}

Systemic lupus erythematosus (SLE) is a multifaceted and complex condition with variable phenotypes and clinical manifestations and a relapsing-remitting course. It is acknowledged that early recognition of SLE can be beneficial for long-term outcomes, allowing early intervention and reducing damage accrual (1). New therapies for SLE offer the opportunity to prevent serious sequelae, and limiting inclusion to those with long-standing disease may underestimate the effectiveness of a new treatment, as late-stage disease may be more difficult to treat and or irreversible (2). Since accurate classification is a prerequisite for including SLE patients in therapeutic trials, the difficulty in classifying early SLE patients may present a limitation to the conduct of clinical and translational studies on early disease.

Because SLE onset is often insidious, with clinically evident disease developing over years, the diagnosis and the classification of SLE may be delayed (3). Both the ACR classification criteria (ACR 1982 (4), ACR 1997 (5)) and the Systemic Lupus International Collaborating Clinics (SLICC) classification criteria (SLICC 2012 (6)) for SLE have lower sensitivity in early disease as compared to established disease (7). Ines et al. reported a higher sensitivity (94\%) for SLICC 2012 compared to ACR 1997 (86\%). Importantly, while the gap between the sensitivity of SLICC 2012 and ACR 1997 was maximal for patients with $\leq 5$ years of SLE duration $(89 \%$ and $76 \%$ respectively) and decreased with longer SLE duration from diagnosis, both sets of criteria performed suboptimally in the first years.

Despite differences in their aims and means, classification and diagnostic criteria both enhance our ability to accurately identify and recognize SLE (8). The aim of the current multicenter study was to evaluate the characteristics of patients with early SLE compared to non-SLE patients, to inform the development of new classification criteria which would potentially and accurately identify more patients in early stages of SLE. Evaluation of the performance of conventional classification criteria in early SLE against the diagnosis made by the rheumatologists was also evaluated.

\section{Patients and methods}

Patients. Seven academic centers in Asia (Manila), Europe (Berlin, Pisa), North America (Boston, Chicago, Toronto) and South America (São Paulo), with experience in the diagnosis and management of SLE, took part in the study. Patients from a multicenter cohort collected by "the Study Group on Early SLE of the Italian Society of Rheumatology" were also included in the study. Participating centers were asked to collect clinical and serological manifestations of subjects with early SLE (group 1) and of subjects with conditions mimicking SLE (group 2, non-SLE) at disease onset. 
Patients had been referred to these centers for evaluation of possible SLE within the previous three years. Early SLE was diagnosed by experienced rheumatologists, based on clinical experience and judgement, and patients did not necessarily fulfill existing classification criteria. Subjects with nonSLE were those referred in the same period with the suspicion of SLE, who ultimately did not receive a diagnosis of SLE by the center's experienced rheumatologists. Non-SLE conditions included infections, hematological diseases (such as lymphoma), other defined connective tissue diseases (Sjögren's syndrome, primary antiphospholipid syndrome, mixed connective tissue disease, systemic sclerosis), other rheumatic diseases (early rheumatoid arthritis), autoimmune diseases (anti-nuclear antibody [ANA]-positive thyroiditis, autoimmune hepatitis, interstitial lung disease) and fibromyalgia. Patients with undifferentiated connective tissue diseases (UCTD) and a follow-up of at least three years were also included in this group.

Data collection. A standardized data extraction form with the ACR 1997, SLICC 2012 and an additional list of 30 items including clinical and serological manifestations attributable to systemic autoimmune diseases, was developed (Appendix 1). Subjects' medical records were reviewed and investigators were requested to add to the list of items any other presenting manifestation that they considered relevant for the diagnosis.

Within the study, standardized definitions for the clinical symptoms (e.g. for pleuritis, alopecia, etc.) were not provided as this study aimed at collecting real-life data. Physicians were asked to report only manifestations that were attributable to possible SLE, after excluding other explanations (e.g. fever in the presence of infection) as far as clinically feasible. Similarly, no specific requirements were made for autoantibody testing assays; negative results reported in clinical charts were also recorded.

Operating characteristics of conventional criteria in early disease. The performance characteristics of the ACR 1997 and SLICC 2012 were evaluated compared to the gold standard of the diagnosis made by the lupus center rheumatologists in terms of accuracy, sensitivity, specificity, positive predictive value (PPV) and negative predictive value (NPV) and their $95 \%$ confidence intervals $(\mathrm{CI})$.

Statistical analysis. Demographic and clinical characteristics of SLE cases and non-SLE mimickers were tabulated. The proportion of subjects with each clinical and laboratory manifestation were calculated. The distribution of variables were compared in early SLE patients and non-SLE mimickers using $\chi^{2}$ or Fisher's exact test. All analyses were performed using Stata 12 (Stata Corp) and $\mathrm{R}$ version 3.2 ; in descriptive statistics, $\mathrm{p}$-values $<0.05$ were considered to be statistically significant. 


\section{Results}

A total of 616 cases were collected, 389 with early SLE and 227 with mimicking conditions. The non-SLE conditions were UCTD (136 patients, 59.9\%), Sjögren's syndrome ( $\mathrm{n}=21,9.3 \%)$, systemic sclerosis $(n=11,4.8 \%)$, primary Raynaud's phenomenon $(n=10,4.4 \%)$, fibromyalgia $(n=8,3.5 \%)$, thyroiditis with positive ANA $(n=7,3.1 \%)$, rheumatoid arthritis $(n=6,2.6 \%)$, mixed connective tissue disease $(n=4,1.8 \%)$, hematological diseases $(n=2)$, infections $(n=2)$, one case each of autoimmune hepatitis and psoriatic arthritis, and 18 miscellaneous diagnoses including rosacea, osteoarthritis, and erythema nodosum.

Demographic data on patients are shown in Table 1. The female to male ratio was higher $(\mathrm{p}<0.001)$ among mimicking conditions, while age at first diagnosis was significantly lower $(\mathrm{p}=0.011)$ among early SLE subjects (Table 1).

\section{Manifestations of early SLE}

ACR and SLICC criteria items were significantly more frequently observed among early SLE patients than mimicking conditions (Table 2). Seizures were uncommon at disease onset, reported in $11(2.8 \%)$ SLE patients, and in no patients with mimicking conditions ( $p=0.009)$. No early SLE subjects presented with peripheral neuropathy. Stroke and myocardial infarction occurred in SLE patients only, but were uncommon $(n=4,1.0 \%$ and $n=3,0.8 \%$, respectively). Unexplained fever was significantly more common in SLE than patients with mimicking conditions (34.5\% vs. 13\%, $\mathrm{p}<0.001)$. Additional differentiating items between SLE patients and patients with mimicking conditions were alopecia $(30.6 \%$ vs. $11.9 \%, \mathrm{p}<0.001)$, weight loss $(13.1 \%$ early SLE vs. $4.4 \%$, $\mathrm{p}<0.001)$, and ascites $(3.1 \%$ vs. $0 \%, \mathrm{p}=0.005)$, respectively.

Among the symptoms that were significantly different between early SLE and mimickers, some were more common in mimicking conditions than in SLE. Among these were Raynaud's phenomenon (22.1\% in SLE vs. $48.5 \%$ in mimicking conditions, $\mathrm{p}<0.001)$, sicca symptoms $(4.4 \%$ vs. $34.4 \%$, p 0.001$)$, dysphagia $(0.3 \%$ vs. $6.2 \%, \mathrm{p}<0.001)$, and fatigue $(28.3 \%$ vs. $37.0 \%, \mathrm{p}=0.024)$. Rashes outside the typical SLE spectrum such as skin vasculitis, were also slightly more frequent among patients with mimicking conditions than SLE subjects (5.9\% in SLE vs. 11.9\%, p=0.009).

\section{Serological and laboratory findings}

Serologies and cytopenias at disease onset are reported in Table 3. Only two early SLE patients $(0.5 \%)$ were ANA negative at disease onset. One patient had a completely negative autoantibody panel, the second had positive anti-Sm and anti-dsDNA antibodies with negative ANA testing. 
Although positive ANA were the most common reason for referral of patients with mimicking conditions, $11(4.9 \%)$ of the patients with mimicking conditions tested negative for ANA at a cutoff of 1:80. Compared to patients with mimicking conditions, patients with early SLE were much more likely to have antibodies to dsDNA (71.7\% vs. $6.9 \%$ of non-SLE) and Sm (30.2 vs. 2.6\%). Anticardiolipin IgM and anti-beta 2 glycoprotein-I antibodies were also more frequent in early SLE, as were positive Coomb's tests, autoimmune hemolytic anemia, hypocomplementemia and leukopenia (Table 3). Antibodies to Ro (SS-A) and La (SS-B) were not differentiating between early SLE (33.2\% and 15.1\% anti-Ro and anti-La positive, respectively) and mimicking conditions (25.6\% and $9.9 \%$, respectively). Thrombocytopenia was rare and was present in $6.6 \%$ of SLE and $4.8 \%$ of mimicking conditions.

\section{Performance characteristics of conventional criteria}

At diagnosis, sensitivity of the ACR 1997 criteria was $66.1 \%$, compared to $83.5 \%$ for the SLICC 2012 criteria. Of the 132 (33.9\%) early SLE patients not meeting ACR criteria, 89 fulfilled three, and 37 fulfilled two ACR 1997 criteria. Six patients met only one ACR criterion. Of the 64 early SLE patients (16.5\%) who did not meet classification by SLICC 2012 criteria, 39 patients fulfilled 3 criteria, and 19 patients only two criteria. Sensitivity and specificity of ACR 1997 and SLICC 2012 criteria for early diagnosis were calculated with the physician diagnosis as the gold standard.

ACR 1997 criteria showed a specificity of 91.6\%, while the specificity of the SLICC 2012 criteria was $82.4 \%$. Accordingly, the accuracy was $75.5 \%$ for the ACR 1997 and $83.1 \%$ for the SLICC 2012 criteria. (Additional material).

\section{Discussion}

In the present study, we investigated clinical symptoms and serological findings at disease onset in a large multicenter, multi-ethnic cohort of 389 SLE patients newly diagnosed at lupus referral centers, and 227 patients referred for possible SLE who were ultimately given another diagnosis after clinical and serological work-up. We identified items that might inform the development of new classification criteria for SLE, with specific interest in improving sensitivity and specificity for classification of early disease. Non-SLE conditions included undifferentiated and other differentiated connective tissue diseases, a variety of other autoimmune disease, and other diseases ranging from hematological disease to fibromyalgia.

For this study, a large number of potential signs and symptoms were systematically recorded. Descriptive statistical analyses revealed that some symptoms were more prevalent in SLE than in mimicking conditions. As expected, these included standard items of existing classification criteria, 
however, signs and symptoms that are not part of current classification criteria also were associated with early SLE, including fever and weight loss. Non-infectious fever was more prevalent in early SLE than in mimicking conditions (34.5\% vs. $13.7 \%)$.

In our cohort of 616 patients, the ACR 1997 criteria had a sensitivity of $66.1 \%$ and a specificity of $91.6 \%$ and the SLICC 2012 criteria a sensitivity of $83.5 \%$ and a specificity of $82.4 \%(8,10,11)$ for early diagnosis. As a result, 132 patients with a clinical diagnosis of SLE (33.9\%) were not classified as SLE patients according to the ACR 1997 criteria, and 64 (16.5\%) did not fulfill the 2012 SLICC classification criteria. These patients were more likely to present a milder picture characterized by arthritis, hematologic manifestations, malar rash, lymphadenopathy, non-infectious fever, alopecia, positive ANA, anti-dsDNA or anti-phospholipid antibodies. In contrast, 19 and 40 patients would be falsely classified as having SLE by the ACR and SLICC criteria, respectively. The accuracy of the ACR 1997 and the SLICC classification criteria were $75.5 \%$ and $83.1 \%$ respectively.

SLE is a disease characterized by a large variety of autoantibodies, and their production has been shown to increase shortly before disease onset (9). A fundamental decision made in the development of the SLICC criteria was that patients were required to have serological evidence of antibodies or immune complex deposition (6). Within the current SLE classification criteria approach, a meta-analysis of published data showed that positive ANA on HEp2 cells, at a titer of at least 1:80, have 98\% sensitivity for SLE (10). Our cohort results support the idea that ANA positivity might be an important discriminant variable in the assessment of patients with the clinical suspicion of SLE. In fact, at disease onset, only two patients diagnosed with SLE were ANA negative, and one of them apparently falsely negative. In addition to negative ANA, fatigue, dysphagia, Raynaud's phenomenon, and some skin lesions (i.e. purpura and skin vasculitis), especially in serologically-negative patients, are either not useful to distinguish from mimicking conditions, or may steer towards alternative diagnosis. These data also emphasize that the differential diagnosis for SLE is long and requires comprehensive experience with other autoimmune and related diseases. In recent years, several studies have characterized SLE patients in the early phases of the disease, highlighting the importance of non-classification criteria symptoms (11-15).

Recently, Rees et al examined the clinical manifestations of SLE patients at onset to develop a risk prediction model for SLE at the time of referral to their general practitioner, rather than a rheumatologist or lupus expert (11). This study showed that patients with SLE consult their physicians frequently in the 5 years preceding the diagnosis for manifestations such as arthralgias, 
rash, and alopecia. While the median time from clinical presentation to SLE diagnosis was greater than one year, manifestations like thrombocytopenia and nephrotic syndrome were more likely to be associated with acute care management (hospital admission or urgent referral) and an earlier diagnosis of SLE.

Since 1990 different studies have examined clinical manifestations and serologies at SLE onset; among non-criteria symptoms, arthralgias, fever, alopecia, Raynaud's phenomenon, non-hemolytic anemia and lymphadenopathy were the most frequently reported (12-15).

Some disagreement can be found between these studies and our data; presumably, differences in inclusion criteria and disease duration limit the comparability of the results.

Our analysis relied on the treating physician's judgment as the gold standard for diagnosis. To minimize the possibility of erroneous diagnosis, the physicians involved in this study were experts at major academic centers who were familiar with the performance of local laboratories. A clinical diagnosis by an SLE expert has the advantage that additional information-for example, patient sex, race, and age at onset-will be integrated into the diagnostic decision. Relying on expert diagnosis also has the advantage of a clear-cut yes or no answer, which allows for analyzing every single patient case that was submitted, while any adjudication proves would have led to the exclusion of individual patients.

Our study entered patients independent of whether they fulfilled ACR or SLICC SLE classification criteria. In contrast to other cohort studies that enrolled patients upon fulfillment of classification criteria (mainly ACR 1997 criteria), our study design allowed for the inclusion of patients at very early disease onset, even before the accrual of standard classification criteria. This methodology was crucial for identifying variables that could distinguish patients with very early SLE, particularly in the absence of disease specific markers such as lupus nephritis or disease specific skin manifestations or autoantibodies that might develop later in the course of disease in these patients.

One limitation of the study might be the relatively small number of patients with African descent and Hispanics; as these patients might have a different disease expression and severity and our results need further confirmation in these ethnic groups.

In conclusion, we identified features at symptom onset that could help in the identification of early SLE. In addition, limitations of the ACR 1997 and the SLICC 2012 criteria in the accurate classification of early SLE were also identified in this cohort. This study is an element in the item generation phase of an ongoing international effort to devise new SLE classification criteria with a focus on early disease, consecutively informing both the nominal group technique exercise for item reduction and the multivariable decision analysis for item weighting. 



\section{References}

1. Oglesby A, Korves C, Laliberte F, Dennis G, Rao S, Suthoff ED, et al. Impact of early versus late systemic lupus erythematosus diagnosis on clinical and economic outcomes. Appl Health Econ Health Policy. 2014;12(2):179-90.

2. Touma Z, Gladman DD. Current and future therapies for systemic lupus erythematosus: Obstacles and recommendations for the development of novel treatments Lupus Sci Med. 2017; In press.

3. Urowitz MB, Gladman DD, Ibanez D, Sanchez-Guerrero J, Romero-Diaz J, Gordon C, et al. American College of Rheumatology criteria at inception, and accrual over 5 years in the SLICC inception cohort. J Rheumatol. 2014;41(5):875-80.

4. Tan EM, Cohen AS, Fries JF, Masi AT, McShane DJ, Rothfield NF, et al. The 1982 revised criteria for the classification of systemic lupus erythematosus. Arthritis Rheum. 1982;25(11):1271-7.

5. Hochberg MC. Updating the American College of Rheumatology revised criteria for the classification of systemic lupus erythematosus. Arthritis Rheum. 1997;40(9):1725.

6. Petri M, Orbai AM, Alarcon GS, Gordon C, Merrill JT, Fortin PR, et al. Derivation and validation of the Systemic Lupus International Collaborating Clinics classification criteria for systemic lupus erythematosus. Arthritis Rheum. 2012;64(8):2677-86.

7. Ines L, Silva C, Galindo M, Lopez-Longo FJ, Terroso G, Romao VC, et al. Classification of Systemic Lupus Erythematosus: Systemic Lupus International Collaborating Clinics Versus American College of Rheumatology Criteria. A Comparative Study of 2,055 Patients From a Real-Life, International Systemic Lupus Erythematosus Cohort. Arthritis Care Res (Hoboken). 2015;67(8):1180-5.

8. Aringer M, Dorner T, Leuchten N, Johnson SR. Toward new criteria for systemic lupus erythematosus-a standpoint. Lupus. 2016;25(8):805-11.

9. Arbuckle MR, McClain MT, Rubertone MV, Scofield RH, Dennis GJ, James JA, et al. Development of autoantibodies before the clinical onset of systemic lupus erythematosus. $\mathrm{N}$ Engl J Med. 2003;349(16):1526-33.

10. Leuchten N, Hoyer A, Brinks R, Schoels M, Schneider M, Smolen J, et al. Performance of Anti-nuclear Antibodies for Classifying Systemic Lupus Erythematosus: a Systematic 
Literature Review and Meta-regression of Diagnostic Data. Arthritis Care Res (Hoboken). 2017.

11. Rees F, Doherty M, Lanyon P, Davenport G, Riley RD, Zhang W, et al. Early Clinical Features in Systemic Lupus Erythematosus: Can They Be Used to Achieve Earlier Diagnosis? A Risk Prediction Model. Arthritis Care Res (Hoboken). 2017;69:833-41.

12. Cervera R, Khamashta MA, Font J, Sebastiani GD, Gil A, Lavilla P, et al. Systemic lupus erythematosus: clinical and immunologic patterns of disease expression in a cohort of 1,000 patients. The European Working Party on Systemic Lupus Erythematosus. Medicine (Baltimore). 1993;72(2):113-24.

13. 2. Pons-Estel BA, Catoggio LJ, Cardiel MH, Soriano ER, Gentiletti S, Villa AR et al. The GLADEL multinational Latin American prospective inception cohort of 1,214 patients with systemic lupus erythematosus: ethnic and disease heterogeneity among "Hispanics". Medicine (Baltimore). 2004;83:1-17.

14. Sebastiani GD, Prevete I, Piga M, Iuliano A, Bettio S, Bortoluzzi A, et al. Early Lupus Project - A multicentre Italian study on systemic lupus erythematosus of recent onset. Lupus. 2015;24:1276-82.

15. Canora J, García M, Mitjavila F, Espinosa G, Suárez S, González-León R et al. Clinical characteristics during diagnosis of a prospective cohort of patients with systemic lupus erythematosus treated in Spanish Departments of Internal Medicine: The RELES study. Rev Clin Esp. 2017 217:7-14 


\section{Contribution}

All authors contributed in the data collection, analyses and interpretation of the data, and in the manuscript writing.

\section{Acknowledgement}

This project has been jointly funded by EULAR and ACR.

Dr. Johnson is supported by a Canadian Institutes of Health Research New Investigator Award.

Dr Touma is supported by the Arthritis Society Young Investigator Award.

Dr. Tedeschi receives salary support from the Lupus Foundation of America Career Development Award.

Rosalind Ramsey-Goldman is the Solovy Arthritis Research Society Professor of Medicine and is supported by Arthritis Research Society at Northwestern University.

We Acknowledge the "Gruppo di Studio on Early SLE" of the Italian Society of Rheumatology for sharing their clinical cases. 
Table 1. Clinical and epidemiological characteristics of the enrolled patients

\begin{tabular}{|c|c|c|c|c|}
\hline & $\begin{array}{c}\text { SLE } \\
(n=389,63.1 \%) \\
\end{array}$ & $\begin{array}{c}\text { Non-SLE } \\
\text { Mimicking } \\
\text { conditions } \\
(\mathbf{n}=\mathbf{2 2 7}, \mathbf{3 6 . 9 \%})\end{array}$ & Total $(n=616)$ & $\begin{array}{c}P \text { - } \\
\text { value }\end{array}$ \\
\hline Female & $345(88.9 \%)$ & $220(96.9 \%)$ & $565(91.9 \%)$ & $<0.001$ \\
\hline Age at first symptoms, & $31.4 \pm 12.3$ & $33.9 \pm 13.5$ & $32.3 \pm 12.7$ & 0.011 \\
\hline \multicolumn{5}{|l|}{ Ethnicity: } \\
\hline Caucasian & $212(54.5 \%)$ & $203(89.4 \%)$ & $415(67.7 \%)$ & \multirow{6}{*}{$-<0.001$} \\
\hline Asian & $113(29.0 \%)$ & $14(6.2 \%)$ & $127(20.7 \%)$ & \\
\hline African descent & $30(7.7 \%)$ & $6(2.6 \%)$ & $36(5.9 \%)$ & \\
\hline Native American & $1(0.3 \%)$ & 0 & $1(0.2 \%)$ & \\
\hline Other & $7(1.8 \%)$ & 0 & $7(1.1 \%)$ & \\
\hline Unknown & $26(6.7 \%)$ & $4(1.8 \%)$ & $27(4.4 \%)$ & \\
\hline
\end{tabular}


Table 2. Clinical manifestations at disease onset in early SLE patients and mimickers

\begin{tabular}{|c|c|c|c|}
\hline & $\begin{array}{c}\text { SLE } \\
(n=389,63.1 \%)\end{array}$ & $\begin{array}{c}\text { Mimickers }(n=227, \\
36.9 \%)\end{array}$ & P-value \\
\hline Fever & $134(34.5)$ & $31(13.7)$ & $<0.001$ \\
\hline Fatigue & $110(28.3)$ & $84(37.0)$ & 0.02 \\
\hline Weight Loss & $51(13.1)$ & $10(4.4)$ & $<0.001$ \\
\hline Malar rash & $193(49.6)$ & $14(6.2)$ & $<0.001$ \\
\hline Subacute cutaneous lupus & $9(2.3)$ & $8(3.5)$ & 0.37 \\
\hline Discoid lesions & $36(9.3)$ & $11(4.9)$ & 0.04 \\
\hline Other rash & $23(5.9)$ & $27(11.9)$ & 0.009 \\
\hline Photosensitivity & $123(31.6)$ & $42(18.5)$ & $<0.001$ \\
\hline Oral Ulcers & $84(21.6)$ & $12(5.3)$ & $<0.001$ \\
\hline Alopecia & $119(30.6)$ & $27(11.9)$ & $<0.001$ \\
\hline Skin Ulcers & $8(2.1)$ & $3(1.3)$ & 0.75 \\
\hline Telangiectasias & $4(1.0)$ & $5(2.2)$ & 0.30 \\
\hline Inflammatory Arthritis & $224(57.6)$ & $60(26.4)$ & $<0.001$ \\
\hline Arthralgias & $79(20.3 \%)$ & $97(42.7 \%)$ & 0.001 \\
\hline Pleuritis & $87(22.4)$ & $6(2.6)$ & $<0.001$ \\
\hline Pericarditis & $73(18.8)$ & $7(3.1)$ & $<0.001$ \\
\hline Ascites & $12(3.1)$ & $0(0)$ & 0.005 \\
\hline Kidney involvement* & $51(13.1)$ & $0(0)$ & $<0.001$ \\
\hline Dry Eyes & $15(3.9)$ & $63(27.8)$ & $<0.001$ \\
\hline Dry Mouth & $14(3.6)$ & $67(29.5)$ & $<0.001$ \\
\hline Dysphagia & $1(0.3)$ & $14(6.2)$ & $<0.001$ \\
\hline Pneumonia & $6(1.5)$ & $0(0)$ & 0.09 \\
\hline Alveolar hemorrhage & $2(0.5)$ & $0(0)$ & 0.53 \\
\hline Pulmonary fibrosis & $2(0.5)$ & $3(1.3)$ & 0.36 \\
\hline Pulmonary hypertension & $5(1.3)$ & $5(2.2)$ & 0.51 \\
\hline Valvular disease & $1(0.3)$ & $0(0)$ & 1.00 \\
\hline Myocardial Infarction & $3(0.8)$ & $0(0)$ & 0.30 \\
\hline Thrombosis & $14(3.6)$ & $2(0.9)$ & 0.06 \\
\hline Swollen fingers & $14(3.6)$ & $11(4.9)$ & 0.52 \\
\hline Raynaud & $86(22.1)$ & $110(48.5)$ & $<0.001$ \\
\hline Livedo reticularis & $12(3.1)$ & $11(4.9)$ & 0.27 \\
\hline Stroke & $4(1.0)$ & $0(0)$ & 0.30 \\
\hline Transitory ischemic attack & $1(0.3)$ & $1(0.4)$ & 1.00 \\
\hline Cognitive impairment & $6(1.5)$ & $1(0.4)$ & 0.43 \\
\hline Seizures & $11(2.8)$ & $0(0)$ & 0.009 \\
\hline Psychosis & $4(1.0)$ & $2(0.9)$ & 1.00 \\
\hline Migraine & $10(2.6)$ & $5(2.2)$ & 1.00 \\
\hline Intestinal vasculitis & $3(0.8)$ & $0(0)$ & 0.30 \\
\hline
\end{tabular}

*Kidney involvement included proteinuria, hematuria, pyuria and casts 
Table 3. Serological abnormalities and autoantibody profiles

\begin{tabular}{|c|c|c|c|}
\hline & $\begin{array}{c}\text { SLE } \\
(n=389,63.1 \%)\end{array}$ & $\begin{array}{l}\text { Mimicking conditions } \\
\quad(n=227,36.9 \%)\end{array}$ & P-value \\
\hline ANA & $387(99.5)$ & $216(95.1)$ & $<0.001$ \\
\hline Anti-dsDNA & $251(71.7)$ & $14(6.9)$ & $<0.001$ \\
\hline Anti-Sm & $90(30.2)$ & $5(2.6)$ & $<0.001$ \\
\hline Anti-Ro & $98(33.2)$ & $53(25.6)$ & 0.06 \\
\hline Anti-La & $41(15.1)$ & $20(9.9)$ & 0.09 \\
\hline Anti-RNP & $85(28.5)$ & $12(5.9)$ & $<0.001$ \\
\hline Anti-Cardiolipin G & $50(18.1)$ & $24(12.1)$ & 0.07 \\
\hline Anti-Cardiolipin M & $36(13.2)$ & $4(2.0)$ & $<0.001$ \\
\hline Lupus anticoagulant & $31(12.7)$ & $27(17.6)$ & 0.17 \\
\hline Anti-beta2-GPI & $30(17.0)$ & $5(4.4)$ & 0.001 \\
\hline Coombs test & $48(12.3)$ & $13(5.7)$ & 0.008 \\
\hline Low complement & $243(73.4)$ & $104(48.4)$ & $<0.001$ \\
\hline Thrombocytopenia & $23(6.6)$ & $10(4.8)$ & 0.37 \\
\hline Leucopenia & $61(16.2)$ & $21(9.8)$ & 0.02 \\
\hline Hemolytic anemia & $18(4.6)$ & $1(0.4)$ & 0.003 \\
\hline
\end{tabular}


Additional material

Table 4. Sensitivity and specificity of the ACR 1997 and SLICC 2012 classification criteria (4a) and operating characteristics results (4b).

a)

\begin{tabular}{|c|c|c|c|}
\hline \multicolumn{4}{|c|}{ ACR 1997} \\
\hline Fulfilled ACR 1997 criteria & $\begin{array}{c}\text { Mimicking } \\
\text { conditions }(\mathrm{n}=227)\end{array}$ & $\operatorname{SLE}(n=389)$ & Total $(n=616)$ \\
\hline No & $208(91.6 \%)$ & $132(33.9 \%)$ & $340(55.2 \%)$ \\
\hline Yes & $19(8.4 \%)$ & $257(66.1 \%)$ & $276(44.8 \%)$ \\
\hline Total & $227(100 \%)$ & $389(100 \%)$ & $616(100 \%)$ \\
\hline \multicolumn{4}{|c|}{ SLICC 2012} \\
\hline Fulfilled SLICC 2012 criteria & $\begin{array}{c}\text { Mimicking } \\
\text { conditions }(n=227)\end{array}$ & SLE $(n=389)$ & Total $(n=616)$ \\
\hline No & $187(82.4 \%)$ & $64(16.5 \%)$ & $251(40.7 \%)$ \\
\hline Yes & $40(17.6 \%)$ & $325(83.5 \%)$ & $365(59.3 \%)$ \\
\hline Total & $227(100 \%)$ & $389(100 \%)$ & $616(100 \%)$ \\
\hline
\end{tabular}

b)

\begin{tabular}{|l|c|c|}
\hline $\begin{array}{l}\text { Operating } \\
\text { characteristics }\end{array}$ & $\begin{array}{c}\text { ACR 1997 } \\
(\mathbf{9 5 \%} \text { CI) }\end{array}$ & $\begin{array}{c}\text { SLICC 2012 } \\
\text { (95\% CI) }\end{array}$ \\
\hline Accuracy & $75.5 \%(71.9-78.8 \%)$ & $83.1 \%(79.9-85.9 \%)$ \\
\hline Sensitivity & $66.1 \%(61.1-70.7 \%)$ & $83.5 \%(79.4-87.0 \%)$ \\
\hline Specificity & $91.6 \%(87.0-94.8 \%)$ & $82.4 \%(76.7-87.0 \%)$ \\
\hline PPV & $93.1 \%(89.3-95.7 \%)$ & $89.0 \%(85.3-92.0 \%)$ \\
\hline NPV & $61.2 \%(55.7-66.3 \%)$ & $74.5 \%(68.6-79.7 \%)$ \\
\hline False positive rate & $3.1 \%(0.2-0.5 \%)$ & $6.5 \%(4.7-8.8 \%)$ \\
\hline False negative rate & $21.4(18.3-24.9 \%)$ & $10.4 \%(8.1-12.1 \%)$ \\
\hline
\end{tabular}


